Canadian Journal of Higher Education Revue canadienne d'enseignement supérieur

Volume 42, No. 1, 2012, pages 43 - 62

\title{
Une enquête sur l'éthique professionnelle des enseignants du collégial québécois : caractéristiques, points de repère et stratégies utilisés pour traiter de préoccupations éthiques
}

Luc Desautels

Cégep régional de Lanaudière à L'Assomption

Christiane Gohier

Université du Québec à Montréal

Jacques Joly, France Jutras et Jean Gabin Ntebutse

Université de Sherbrooke

\section{RÉSUMÉ}

Certains résultats d'une enquête sur l'éthique professionnelle menée auprès d'enseignants des collèges québécois sont comparés avec une recension des écrits sur la question et les résultats d'entrevues réalisées préalablement dans des groupes de discussion. Tant au sujet des indices ou des raisons démontrant le caractère éthique de certaines préoccupations professionnelles que sur les points de repère et les stratégies de résolution privilégiés, une grande convergence est observée entre les répondants à l'enquête, les participants aux groupes de discussion et les constats qui émergent de la recension des écrits. Dans l'ensemble, les raisons évoquées par les enseignants pour affirmer le caractère éthique de certaines préoccupations professionnelles renvoient surtout aux cas de conscience et aux conflits de valeurs ainsi qu'à l'anticipation 
de conséquences sur autrui, particulièrement vis-à-vis des étudiants. Seule la raison « mise en cause de l'intégrité professionnelle » n'avait pas été relevée dans les études antérieures. Les points de repère principaux auxquels se fient les enseignants sont les textes normatifs adoptés par les établissements et leurs valeurs et principes personnels. Ces résultats suggèrent qu'il existe un équilibre, chez les enseignants, dans l'utilisation de ces deux types de repères, externes et internes. Enfin, les principales stratégies de résolution des dilemmes éthiques déployées par les enseignants consistent essentiellement en la discussion entre pairs et la réflexion personnelle.

\begin{abstract}
Some results of a survey on professional ethics conducted among francophone college teachers in Quebec are compared with a review of the literature and the results of focus groups previously held on the subject. The justifications reasons behindof the ethical nature of certain professional concerns, along with the guidelines and the resolution strategies used to address those concerns, are found to be similar. These justifications relate primarily to situations where there is a conflict of conscience, a conflict of values or consequences on others, particularly students; the calling into question of professional integrity stands out as a particular justification not mentioned in earlier studies. The guidelines to deal with dilemmas are also similar: institutional normative texts and values deemed relevant by the teachers come first. The results show a good balance between the use of these two types of guidelines (external and internal) by teachers. The main strategies applied by teachers for resolving dilemmas are peer discussions and personal reflection.
\end{abstract}

\title{
INTRODUCTION
}

Dans le cadre d'une recherche mixte entreprise pour mettre en évidence les préoccupations éthiques des enseignants du collégial (Gohier, Jutras et Desautels, 2007a), le point de vue d'enseignants du réseau collégial québécois francophone, privé et public, a été sollicité afin d'identifier leurs préoccupations éthiques, les indices ou les raisons leur permettant de reconnaître le caractère éthique de ces préoccupations ainsi que les points de repère et les stratégies utilisés pour les traiter. Dans la première phase de cette recherche, une série de groupes de discussion (focus groups) menés en 2006-2007 (Gohier, Jutras et Desautels, 2007b) a permis une première exploration de ces questions et les résultats (Desautels, Gohier et Jutras, 2009; Gohier, Desautels et Jutras, 2010a) ont servi à élaborer un questionnaire distribué en ligne à plus de 8000 enseignants du réseau collégial, au printemps 2008 (Gohier, Desautels, Joly, Jutras, Ntebutse, 2010b). L'objectif du présent article est de comparer certains résultats obtenus lors de cette enquête avec les constats qui émergent d'une recension des écrits scientifiques, d'une part, et avec les résultats obtenus dans le cadre des groupes de discussion, d'autre part. Il s'agit plus précisément d'examiner si les indices, ou raisons, évoqués par les répondants pour reconnaître le caractère éthique de certaines préoccupations professionnelles, ainsi que les points de 
repère et les stratégies de résolution qu'ils utilisent, sont semblables ou non à ceux relevés dans la recension d'écrits et à ceux dégagés dans les groupes de discussion. Après avoir succinctement rappelé la problématique d'ensemble de la recherche, le cadre théorique particulier est développé, puis la méthodologie propre à l'enquête par questionnaire est présentée, et les résultats pertinents divulgués, comparés et discutés.

\section{LA PROBLÉMATIQUE D'ENSEMBLE}

Les conceptions de l'éthique sont très diversifiées et la parenté de celle-ci avec la morale, toutes deux se rapportant étymologiquement aux termes ethos ou mores, renvoie à plus de deux millénaires de réflexion dans la pensée philosophique occidentale. Dans les deux cas, les mœurs, le comportement, le rapport à l'autre sont visés. Par ailleurs, la morale est habituellement considérée comme plus normative, socialement marquée, tandis que l'éthique est considérée comme plus réflexive, par une appropriation et une mise à distance par l'individu de la morale prescrite, en fonction de ses valeurs et de son expérience (Gohier, 2005). Dans le cadre du projet de recherche sur lequel s'appuie le présent article, qui met l'accent sur le point de vue des enseignants, le concept " d'éthique » a été retenu, car il laisse la place à l'expérience individuelle et à la réflexivité. Pour rester dans un registre contemporain, c'est la définition de l'éthique proposée par Ricœur (1991), l'un des chefs de file de la réflexion dans ce domaine, qui a été privilégiée. Ricœur, se réclamant de l'héritage aristotélicien, définit l'éthique comme une visée, celle « de la vie bonne, avec et pour les autres, dans des institutions justes » (1991, p. 257). Cette définition peut être complétée par celle de Beaudoin s'inspirant d'Auroux (1990, cité dans Baudouin, 1994, p. 20) comme "l'étude théorique des principes qui guident l'action humaine dans les contextes où le choix est possible ». Dans le premier cas, on s'interroge sur le comportement à adopter et les actions à poser pour atteindre l'idéal de la vie bonne; dans l'autre, on examine les principes qui guident l'action dans un questionnement où les recommandations pour l'action sont déduites de principes éthiques. Dans les deux cas, il s'agit d'une activité réflexive au regard de son comportement envers l'autre.

Plusieurs auteurs mettent l'accent sur la nécessité pour les professionnels de l'enseignement de prendre en compte la dimension éthique de l'acte éducatif. Ainsi, dans le monde anglophone, la recension d'écrits des deux dernières décennies menée par Campbell (2008) montre qu'il existe un consensus quant à la nature éthique du travail de l'enseignant, que ce travail commande une véritable éthique professionnelle et que cette éthique professionnelle doit répondre aux défis que pose l'enseignement, et particulièrement aux dilemmes qu'y rencontrent les enseignants. En contexte francophone, Prairat (2005), Moreau (2007) ainsi que Desaulniers et Jutras (2006) font des affirmations semblables. Par exemple, Moreau (2003) se propose de montrer, dans sa thèse de doctorat, " que l'éthique des enseignants est une éthique professionnelle et non une éthique privée, que cette éthique professionnelle est un trait essentiel de la professionnalité (sic) des enseignants, et peut-être en est-il le trait structurant, que cette éthique professionnelle est le résultat d'une construction [...] » (p. 3-4). C'est dans cette perspective tout à fait cohérente avec celle de l'éthique appliquée, entendue non seulement comme éthique sectorielle, mais aussi comme démarche principalement inductive et interactive qui réserve le rôle premier aux acteurs (Parizeau, 1994; Desautels, 2005), que s’inscrivent les résultats de la recherche ici présentés. 
Peu de recherches empiriques ont été réalisées à ce jour pour recueillir le point de vue d'enseignants sur leur éthique professionnelle. Du côté francophone, on peut citer l'enquête réalisée par Jutras, Joly, Legault et Desaulniers (2005) sur les conceptions de la profession des enseignants du primaire et du secondaire au Québec, enquête dont certains éléments touchent la question de l'éthique professionnelle. Ces auteurs rapportent un modèle dominant d'éthique professionnelle chez les enseignants, celui de la conception humaniste, où l'enseignant " [...] se considère comme chargé du développement global des élèves [...]. Il se perçoit comme un aide, appelé à intervenir dans la classe et aussi individuellement auprès de chacun » (p. 280) alors que dans un autre modèle émergent " l'enseignant centre son action sur la qualification de l'élève et le développement de ses compétences [...] et sa conscience professionnelle pourrait facilement se développer dans le cadre d'une éthique professionnelle encadrée ou non par un code d'éthique » (p. 281). Du côté anglophone, Campbell (2008) signale quelques recherches empiriques sur l'éthique professionnelle des enseignants ayant été réalisées depuis 20 ans, celles de Buzzelli et Johnston (2002), de Campbell (2003), de Jackson, Boostrom et Hansen (1993) et de Richardson et Fenstermacher (2001). Aux dires de Campbell (2008), ces recherches sont principalement de nature qualitative et leurs données servent surtout à illustrer la pertinence du questionnement éthique pour les enseignants et, à l'occasion, à soutenir un point de vue philosophique sur le sujet. D'après Hansen (2001), la recherche sur le terrain, en classe ou dans les établissements, n'en est qu'à ses débuts. En ce sens, le présent article contribue à l'accroissement du corpus des recherches empiriques dévolues à l'éthique professionnelle de l'enseignement et elle table sur le point de vue des enseignantes et des enseignants consultés.

\section{LE CADRE THÉORIQUE}

Aux fins du présent article, à défaut de pouvoir compter sur un riche corpus de recherches empiriques, sont considérés d'autres types d'écrits sur le sujet, à savoir les essais philosophiques et les textes destinés à la formation des enseignants. Même s'ils ne sont pas le fruit d'une enquête scientifique sur le terrain, leurs auteurs peuvent bien souvent tabler sur leur propre expérience d'enseignement et sur des cas réels rencontrés lors de la formation d'autres enseignants. En incluant ce matériel, l'inventaire des préoccupations éthiques, des indices ou des raisons leur permettant de reconnaître le caractère éthique de ces préoccupations ainsi que les points de repère et les stratégies utilisés pour les traiter, devient plus consistant. L'ensemble des écrits recensés ici servira à établir la comparaison entre les réponses obtenues dans notre enquête et les travaux existants.

Ainsi, quant aux indices et aux raisons qui pourraient amener un enseignant à prendre conscience de la dimension éthique de son travail, si Watson (2007) avance qu'une sorte de contrat moral lie l'enseignant à la société, il en découle une série d'obligations non seulement afférentes aux tâches d'enseignement proprement dites, mais aussi d'engagement dans la vie administrative de l'institution et dans le service à la collectivité. On peut en déduire que les tiraillements ou les manquements face à ces obligations constitueront autant d'occasions de prendre conscience de la dimension éthique tant pour l'acteur lui-même que pour ses collègues qui l'observent. Il en irait ainsi d'ailleurs de ceux qui travaillent dans un établissement ayant adopté un code d'éthique ou de déontologie adapté à l'enseignement, par exemple celui de la University of Southern California 
(2004, cité par Couch et Dodd, 2005). De même, à défaut de développer certaines vertus particulièrement pertinentes pour l'enseignant (McInthyre, repris par McFarlane, 2004) comme le respect, la sensibilité, la fierté, le courage, l'équité, l'ouverture d'esprit, la retenue et la collégialité, rencontrer des situations où l'on manque de l'une ou l'autre peut susciter la réflexion éthique.

Un autre révélateur de la prise de conscience de l'éthique dans le travail de l'enseignant a trait aux dilemmes qui surviennent lorsque surgit un conflit de valeurs (Mirk, 2005) ou, comme l'ont constaté les chercheurs suédois Norberg et Johansson (2007), lorsque ces conflits mettent aux prises les valeurs de la profession et les valeurs personnelles des individus, quand les droits des élèves sont en jeu. Que ces dilemmes soient définis tels des choix difficiles entre deux ou plusieurs possibilités également acceptables (Nash, 1996), entre des possibilités également inacceptables (Young, 1995) ou comme un choix d'action qui implique de faire mal en vue de générer le bien (Boss, 1998), il est clair que les enseignants rencontrent des dilemmes éthiques dans leur vie professionnelle (Campbell, 2008). En fait, chaque fois qu'un acte entraîne ou peut entraîner des conséquences négatives sur autrui (Brunh, Zajac, Al-Kazemi et Prescott, 2002), la conscience professionnelle est convoquée. Face à de telles situations, insatisfaits des solutions techniques ou de leurs manières spontanées d'agir, les enseignants tentent alors, « par la délibération rationnelle, de produire des normes valides capables de guider leurs futures décisions » (Moreau, 2003, p. 62).

En somme, il est fréquent qu'un enseignant prenne conscience de la dimension éthique de son travail à l'occasion d'un manquement, soit de sa part ou dont il est témoin, ou parce qu'il rencontre un dilemme dans sa pratique, dilemme dans lequel se joue un conflit de valeurs, dilemme mettant en cause le bien d'autrui et face auquel la délibération rationnelle est nécessaire. Les raisons évoquées pour identifier une préoccupation éthique par les participants aux groupes de discussion (focus groups) en 2006-2007, lors de la première phase de ce projet de recherche (Gohier et coll., 2010), recoupent en majeure partie celles repérées dans les écrits scientifiques (Mirk, 2005; Norberg et Johansson, 2007; Nash, 1996; Young, 1995; Boss, 1998; Brunh et coll., 2002; Moreau, 2003). Ces raisons renvoient principalement aux valeurs mises en cause, aux cas de conscience vécus et au caractère indécidable de certaines situations ou conflits qui ne trouvent pas de réponse absolue et requièrent l'exercice de la réflexion et du jugement éthiques.

Quant aux points de repère auxquels l'enseignant peut se référer lorsqu'il s'interroge sur son éthique professionnelle ou sur un dilemme qu'il rencontre dans sa pratique, les écrits scientifiques proposent des codes d'éthique ou de déontologie, des principes, des normes, des commandements, des responsabilités, des cadres de référence et des valeurs. Campbell (2000) cite plusieurs exemples de tels codes. Ces différents codes oscillent entre déontologie etéthique, la première se caractérisant par le rappel des devoirs de la profession et la seconde pointant plutôt vers les valeurs qui inspirent l'action professionnelle (Desaulniers, 2007). Par exemple, au Canada, les principes éthiques mis de l'avant par la Society for Teaching and Learning in Higher Education (STLHE) passent en revue, selon une approche déontologique, les devoirs des enseignants dans plusieurs champs tels la compétence disciplinaire, la compétence pédagogique, le traitement des sujets sensibles et le développement des étudiants, alors que les Normes de déontologie de la profession enseignante (2006) publiées par l'Ordre des enseignantes et des enseignants de l'Ontario le font plutôt selon une perspective éthique, en posant les valeurs qui fondent l'action des 
enseignants : l'empathie, le respect, la confiance et l'intégrité. Le contraste entre ces deux textes illustre bien la différence entre déontologie et éthique, même si les appellations choisies par les deux organismes entretiennent la confusion entre les deux termes.

Le débat sur la pertinence des codes d'éthique ou de déontologie en éducation est bien présent dans les écrits scientifiques. Par exemple, lorsque Legault (1997) s’interroge sur la fonction à donner aux « codes d'éthique » dans nos institutions et sur la façon de favoriser l'émergence de codes axés sur des valeurs partagées, Campbell (2000) pourrait répondre en évoquant les trois visées possibles de ces codes. La première visée est de permettre à la profession de se déclarer imputable envers la population et de fournir un cadre punitif et disciplinaire pour les membres. La seconde est de fournir une ressource, un guide pour aider les enseignants qui font face à des cas éthiques dans le cadre de leur travail. La troisième combine les deux premières avec une dominante de l'un ou l'autre des deux aspects. Ces visées semblent toutefois insuffisantes pour générer un cadre de référence éthique nécessaire au développement du "souci éthique » souhaité par Lafortune, Thidodeau, Ticon et Walther (2008). Ce souci éthique, qui caractérise la prise de décision de l'enseignant (Desaulniers, 2007), se rapproche de la sensibilité éthique (ethical awareness) ou de la connaissance éthique (ethical knowledge) qui constitue le signe du professionnalisme enseignant selon Campbell (2003). De toute façon, il existe un consensus sur le fait que si les codes d'éthique peuvent être utiles, ils ne sont jamais suffisants (Campbell, 2008). Il convient en outre de rappeler, avec Moreau (2007, p. 72), qu'« une déontologie ne crée pas la réflexivité, [...] elle la suppose ».

En plus de tous ces codes d'éthique ou de déontologie adoptés par des institutions ou des organismes, on peut ajouter quelques propositions venant des chercheurs eux-mêmes. Ainsi Blunden (1996) dénombre cinq devoirs (loyalties) auxquels serait tenu l'enseignant : envers la discipline, les étudiants, les collègues, l'institution et la société. En cas de conflit entre ces devoirs, il recommande d'appliquer les principes de l'utilitarisme, c'est-à-dire de privilégier la solution qui génère le plus de conséquences positives pour le plus grand nombre de personnes impliquées. Watson (2007) dresse la liste des commandements de l'enseignement supérieur, où l'on trouve notamment l'obligation de toujours s'efforcer de dire la vérité, de respecter les collègues, les étudiants et particulièrement les adversaires, et de ne jamais s'estimer satisfait du travail accompli. Hardy (2002) emprunte à Pratt (1998) ce qu'il appelle les cinq responsabilités du professeur (maintien de la compétence, respect du plan de cours, évitement du plagiat, évaluation cohérente avec ce qui a été enseigné, confidentialité des évaluations) et les cinq droits de l'apprenant (être traité avec respect; s'instruire dans un environnement sécuritaire, juste et productif; être informé des attentes du cours et du programme; avoir accès à l'enseignant et à des ressources; pouvoir questionner les actions et jugements de l'enseignant).

Par ailleurs, Smith (1996) présente quatre normes qui devraient gouverner l'action de l'enseignant dans la relation avec les étudiants : l'honnêteté, le respect de sa parole, le respect des individus, l'équité. Desaulniers et Jutras (2006) relèvent aussi plusieurs composantes éthiques, telles la disponibilité, l'attention aux besoins, ainsi que la maîtrise disciplinaire et pédagogique que l'on peut mettre en relation avec les types d'interventions (techniques, cliniques ou relatives au savoir) effectuées par l'enseignant. Longhi (1998) recommande une déontologie mettant en exergue la probité, l'impartialité et la neutralité dans tous les domaines qui relèvent de l'enseignement. Gohier (2005), se référant à Lenoir 
(1991), rappelle qu'il y a des valeurs universelles, tels le courage, l'honnêteté, la bonté et la miséricorde qui constituent le matériau permettant, par le biais de la discussion, d'arriver à des consensus sur des comportements souhaitables, y compris en éducation. Cette affirmation rejoint le constat de Campbell (2000) à l'égard du fait que, de plus en plus, les auteurs traitant de l'éthique en éducation s'entendent pour dire que la subjectivité éthique et le relativisme moral n'ont pas de place dans les discussions sérieuses portant sur l'éthique professionnelle.

Dans le cadre de la première phase du projet de recherche sur lequel s'appuie le présent article (Gohier et coll., 2010), les participants aux groupes de discussion (focus groups) ont dit utiliser plusieurs points de repère pour résoudre leurs préoccupations éthiques, que l'on peut qualifier d'externes ou d'internes à la personne, points de repère semblables à ceux répertoriés dans les écrits scientifiques. À l'égard des repères externes, ils disent se tourner essentiellement vers les politiques institutionnelles, règles et codes de déontologie (Campbell, 2000); certains participants déplorent par ailleurs l'absence de balises mieux définies. Quant aux points de repère internes, ce sont surtout les principes et valeurs personnels qui sont évoqués (Gohier, 2005; Lenoir, 1991).

Au sujet des stratégies pour traiter des questions d'éthique professionnelle rencontrées par l'enseignant dans sa pratique, des deux tendances identifiées par Fortin (1995), à savoir « l'application de la règle » ou « la discussion et la justification », c'est certainement la seconde avenue qui est privilégiée par les auteurs qui s'intéressent à la question. Ainsi, Lafortune et coll. (2008) soutiennent que c'est dès la formation initiale que l'habitude de la réflexion approfondie doit être prise puisque la simple conformité à des normes édictées par les autres ne peut suffire. Dans la même veine, Strike (1990) suggère que l'accent, dans la formation éthique des enseignants, soit mis sur le développement du jugement et Smith (1996) fait appel à la pratique réflexive développée par Schön et aux études de cas. Même insistance sur le développement de la pensée critique chez Hanson (1996) et sur l'analyse réflexive au ministère québécois de l'Éducation (Gouvernement du Québec, 2001).

Pour sa part, Moreau (2007) recommande d'utiliser une démarche d'éthique appliquée plutôt qu'une éthique de la conviction. Fisch (1996) affirme que la meilleure façon d'apprendre sur les enjeux éthiques de la profession enseignante demeure l'échange entre pairs sur des cas vécus ou fictifs. Toutes ces recommandations se conjuguent avec l'éthique de la discussion promue par Habermas (1991) telle que reprise et adaptée à l'éthique professionnelle par Legault (1999), incluant celle de l'enseignement (Desaulniers, Jutras et Legault, 2005; Gohier, 2005). En fait, ce que Massé et Saint-Arnaud (2003) affirment du domaine de la santé vaut aussi pour le domaine de l'enseignement : «l'éthique de la discussion doit constituer une alternative tant au relativisme paralysant qu'aux tergiversations des situationnistes, à l'arbitraire d'un principisme [sic] mécaniste et au rigorisme sécurisant de la loi » (p. 166). On voit également apparaître cette perspective chez Keith-Spiegel et ses collaborateurs (1996) puisque la réflexion, la consultation de collègues et la discussion avec le fautif constituent des ingrédients clés dans les 13 étapes suggérées pour intervenir auprès d'un collègue dont le comportement manque d'éthique professionnelle. D’après Knight et Auster (1999), s’il est généralement recommandé d'intervenir dans de tels cas, leur enquête menée auprès de 804 professeurs d'universités américaines montre que ce sont habituellement les professeurs les plus expérimentés qui se permettent d'intervenir auprès d'un collègue fautif. 
Pour leur part, les participants aux groupes de discussion tenus en 2006-2007, lors du volet qualitatif de cette étude mixte (Gohier et coll., 2010a), mentionnent des stratégies à base de discussion avec différents acteurs, au premier chef les collègues, ainsi que l'usage de la réflexion et de l'argumentation. Ils insistent sur la difficulté d'engager la discussion avec les collègues sur des questions d'ordre éthique, surtout quand ceux-ci sont directement concernés, conduisant dans certains cas à une réaction de repli ou de neutralité face à ces situations. Dans l'ensemble, les stratégies privilégiées par les participants aux groupes de discussion vont dans le même sens que ce que révèlent les écrits scientifiques investigués (Lafortune et coll., 2008; Strike, 1990; Smith, 1996; Moreau, 2007; Fisch, 1996; Legault, 1999; Desaulniers et coll., 2005; Gohier, 2005).

\section{LA MÉTHODOLOGIE}

La recherche sur laquelle s'appuie le présent article est basée sur un devis mixte exploratoire (Creswell et Plano-Clark, 2007) dont la visée est descriptive. La première phase, qualitative, a été réalisée à l'aide de groupes de discussion (Gohier et coll., 2010a) et la seconde phase, quantitative, à l'aide d'un questionnaire en ligne élaboré à partir des résultats de la première phase (Gohier et coll., 2010b). Le questionnaire comprend six grandes sections : les préoccupations éthiques liées au travail d'enseignant du collégial, les raisons ou indices qui permettent d'identifier une préoccupation d'ordre éthique, les stratégies de résolution des préoccupations éthiques, les points de repère (principes, modèles) qui aident à résoudre des préoccupations éthiques, les moyens susceptibles de favoriser le développement de l'éthique professionnelle des enseignants du collégial et les renseignements sociodémographiques. Une première version du questionnaire a été validée auprès de 20 personnes ayant participé aux groupes de discussion. Des améliorations et des clarifications ont été apportées à la version définitive du questionnaire mis en ligne.

Chaque section comporte une consigne invitant les répondants à choisir parmi un nombre de réponses possibles élaborées à partir des résultats des groupes de discussion. Les répondants doivent indiquer si oui ou non chaque élément représente bien leur opinion (Blais et Durand, 2003). La possibilité leur est aussi donnée d’ajouter un élément ou un commentaire à la fin de chaque section ou bloc d'éléments. Ainsi, six choix de réponses portent sur les raisons qui permettent aux enseignants de reconnaitre une préoccupation d'ordre éthique; neuf choix de réponses visent à identifier les stratégies privilégiées pour résoudre des préoccupations éthiques; enfin, sept choix de réponses portent sur les points de repère, principes ou modèles qui aident à résoudre des préoccupations éthiques. Chacune de ces listes de choix de réponses apparaît dans la légende des histogrammes présentés dans les figures 1,2 et 3.

La mise en ligne du questionnaire a été confiée à une entreprise spécialisée dans ce type d'enquête (Écho sondage). Toutes les personnes ciblées pour constituer l'échantillon ont reçu un courriel personnalisé à leur adresse professionnelle au mois de mars 2008, les invitant à accéder au site dédié pour répondre au sondage dans les meilleurs délais. Deux relances ont été effectuées aux mois d'avril et de mai 2008. Les enseignants devaient d'abord prendre connaissance des visées de la recherche et des mesures prises pour assurer la confidentialité des données, puis donner leur consentement pour finalement pouvoir accéder au questionnaire. 
En 2007, selon les fichiers obtenus du ministère de l'Éducation, du Loisir et du Sport l'ensemble des enseignants permanents du collégial public et des enseignants à temps complet pour le collégial privé au Québec était constitué de 4191 hommes, de 4163 femmes et de 20 autres personnes dont le genre n'est pas précisé. Cette liste nominative comprenait aussi les collèges où travaillaient ces enseignants. Les adresses de courriel de chaque personne de la liste ont été retracées à l'aide des sites web de chacun des collèges. Les raisons qui ont présidé au choix de cibler les enseignants permanents, ou à temps complet, tiennent à la volonté d'obtenir le point de vue de professeurs bien enracinés dans le réseau collégial, d'une part, et facilement joignables, d'autre part.

Chacun de ces 8374 enseignants permanents ou à temps complet, qui enseignaient dans un cégep ou un collège privé au moment de la constitution de la base de données, a reçu la même invitation. De ce nombre, 1345 personnes ont répondu, dont 581 hommes et 764 femmes. Le taux de réponse est de $16 \%$ et, plus particulièrement, il se situe à $14 \%$ au secteur préuniversitaire, à 16,9\% au secteur technique et à 15,4\% en formation générale. Ces taux se comparent aisément aux taux de réponse obtenus dans d'autres études. En effet, dans une méta-analyse effectuée par Shih et Fan (2008), sur 37 études comprenant 39 comparaisons indépendantes entre les enquêtes menées via le web et celles effectuées par la poste, il ressort que 11 études menées via le web ont un taux de réponse inférieur à $20 \%$ et variant de $7 \%$ à $19 \%$. Ces taux de réponse s'inscrivent toutefois dans la tendance à la baisse observée par plusieurs auteurs à l'égard des enquêtes en ligne (Munoz-Leiva, Sanchez-Fernandez, Montoro-Rios et Ibanez-Zapata, 2009; Lozar Manfreda, Bosnjak, Berzelak, Haas et Vehovar, 2008; Sheehan, 2001). Enfin, soulignons que la taille de l'échantillon final est tout de même largement supérieure à celle de l'échantillon obtenu dans le cadre d'une recherche semblable menée par Knight et Auster (1999) auprès d'une population d'enseignants universitaires et qui portait aussi sur des préoccupations relatives à l'éthique professionnelle.

Après avoir retiré cinq répondants dont le questionnaire était demeuré vierge ou incomplet, les données ont été pondérées en fonction du genre et du secteur d'enseignement (général, technique, préuniversitaire) afin de corriger les biais de l'échantillon. Le tableau 1 montre la répartition de l'échantillon en pourcentage, en fonction des variables sexe, âge, secteur d'enseignement et scolarité. On remarque le peu d'influence, et donc le faible biais initial, de la pondération sur la répartition en fonction de l'âge, du secteur d'enseignement et de la scolarité, ce qui suggère que malgré le faible taux de réponse, les caractéristiques de l'échantillon correspondent à la population étudiée.

\section{LES RÉSULTATS}

Les synthèses des résultats sont présentées au moyen d'histogrammes qui mettent en évidence les pourcentages relatifs aux choix de réponses des répondants pour chacun des trois thèmes au cœur de cet article. La figure 1 rend compte des raisons ou des indices qui permettent de reconnaître une préoccupation d'ordre éthique, la figure 2, des points de repère (principes, modèles) qui aident à résoudre des préoccupations éthiques et la figure 3 , des stratégies privilégiées pour résoudre des préoccupations éthiques. Conformément aux constats relevés dans la recension d'écrits et aux résultats des groupes de discussion, les préoccupations éthiques incluent ici les dilemmes éthiques, sans toutefois s'y restreindre. Ce recoupement entre les deux notions est inhérent à notre investigation et est inclus dans notre questionnaire. 
Tableau 1.

Caractéristiques de l'échantillon exprimées en pourcentage avant et après pondération

\begin{tabular}{lcc}
\hline Caractéristiques & $\begin{array}{c}\text { Échantillon non pondéré } \\
\text { n=1 340 }\end{array}$ & $\begin{array}{c}\text { Échantillon pondéré } \\
\mathbf{n}=\mathbf{1} 340\end{array}$ \\
\hline Sexe & $56,8 \%$ & $50,0 \%$ \\
Féminin & $43,2 \%$ & $50,0 \%$ \\
Masculin & & \\
Âge & $0,8 \%$ & $0,8 \%$ \\
$20-29$ & $10,6 \%$ & $10,1 \%$ \\
$30-39$ & $34,0 \%$ & $33,3 \%$ \\
$40-49$ & $49,9 \%$ & $50,8 \%$ \\
$50-59$ & $4,7 \%$ & $4,9 \%$ \\
60 et plus & & \\
Principal secteur d'enseignement & $55,3 \%$ & $53,1 \%$ \\
Technique & $25,3 \%$ & $26,6 \%$ \\
Pré-universitaire & $19,4 \%$ & $20,3 \%$ \\
Formation générale & $3,8 \%$ & \\
Scolarité reconnue aux fins de la rémunération & $3,7 \%$ \\
16 ans et moins & $22,7 \%$ & $21,8 \%$ \\
17 ans & $28,7 \%$ & $28,6 \%$ \\
18 ans & $44,8 \%$ & $45,9 \%$ \\
19 ans et plus &
\end{tabular}

Parmi les raisons ou indices permettant d'identifier une préoccupation éthique et que l'on a proposés aux répondants de l'enquête, trois recueillent l'adhésion du plus grand nombre. En effet, une situation de la vie professionnelle de l'enseignant met en jeu l'éthique si elle suppose une prise de décision ayant des répercussions sur l'intégrité professionnelle de l'enseignant $(85,2 \%)$ ou entraînant des conséquences sur autrui $(78,2 \%)$, ou encore si elle exige une réflexion préalable parce qu'elle se présente comme un cas de conscience $(77,4 \%)$.

Toutefois, les trois autres raisons alléguées sont pointées par bien plus de la moitié des répondants et pour deux de ces raisons, ce sont les deux tiers des répondants qui les évoquent. Ainsi, en ordre décroissant d'adhésion, les répondants reconnaissent le caractère éthique d'une situation quand elle présente un conflit entre les valeurs personnelles et collectives $(68,5 \%)$, quand il y a un conflit de valeurs dans la situation $(67,4 \%)$ ou lorsque la situation confronte l'idéal professionnel de l'enseignant (56,6 \%). Les suggestions des répondants apparaissant dans la rubrique « Autre raison » reprennent ou explicitent celles répertoriées dans le questionnaire. Par exemple, certains ont nommé des valeurs susceptibles d'entrer en conflit avec d'autres, particulièrement le respect et l'équité, ou ont insisté sur les conséquences possibles d'une décision sur l'étudiant ou sur le département. Quelques répondants (10,6 \%) signalent plutôt des indices de la présence 
Figure 1.

Les raisons qui permettent de reconnaître une préoccupation d'ordre éthique

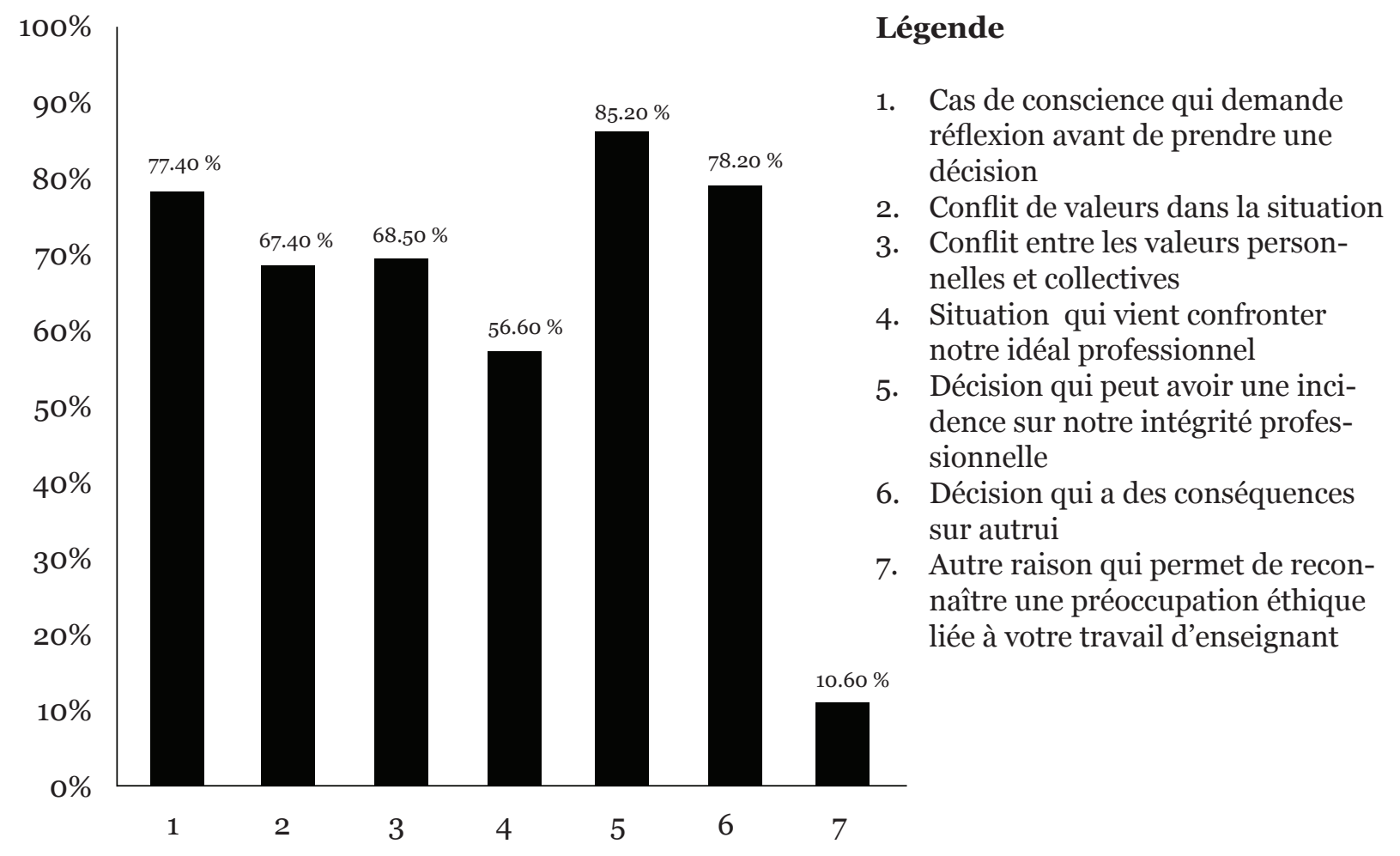

d'un problème éthique tels l'insomnie, le malaise, le déchirement entre deux choix ou le fait d'être déstabilisé par une situation.

Des sept points de repère (principes, modèles) potentiellement utiles pour résoudre des préoccupations (voir la figure 2), deux se démarquent fortement par le grand nombre de répondants qui les retiennent : les principes et les valeurs personnels des enseignants $(88,3 \%)$ ainsi que les politiques et les règlements du collège $(84,1 \%)$, qui seraient utilisés par plus des quatre cinquièmes des répondants.

La convention collective de travail demeure une référence utile pour plus de la moitié des répondants (57,8 \%), alors que les us et coutumes de l'institution ainsi que le code de vie du collège reçoivent l'aval de près de la moitié d'entre eux (respectivement 49,6 \% et $48,9 \%$ ). Le code d'éthique d'une autre profession sert à peu près au tiers des répondants $(29,8 \%)$ et seule une minorité $(5,1 \%)$ a recours à des convictions religieuses pour traiter des questions d'éthique professionnelle. Parmi les autres points de repère suggérés par près de $8 \%$ des répondants, deux ne peuvent être associés aux choix proposés par le questionnaire dans ce thème ou dans un autre. Il s'agit de la référence au « bon sens » et aux différentes chartes de droits et autres lois.

Quant aux stratégies privilégiées pour résoudre des préoccupations éthiques (voir la figure 3), ce sont clairement la discussion avec des collègues (95,9 \%) et la réflexion personnelle $(89,5 \%)$ qui sont le plus mises à contribution selon les répondants à l'enquête.

La discussion apparaît comme une stratégie très largement déployée : si celle avec les collègues domine, les discussions avec les étudiants $(69,6 \%)$, les proches $(56,2 \%)$, les 
Figure 2

Les points de repères (principes, modèles) utilisés pour résoudre des préoccupations éthiques

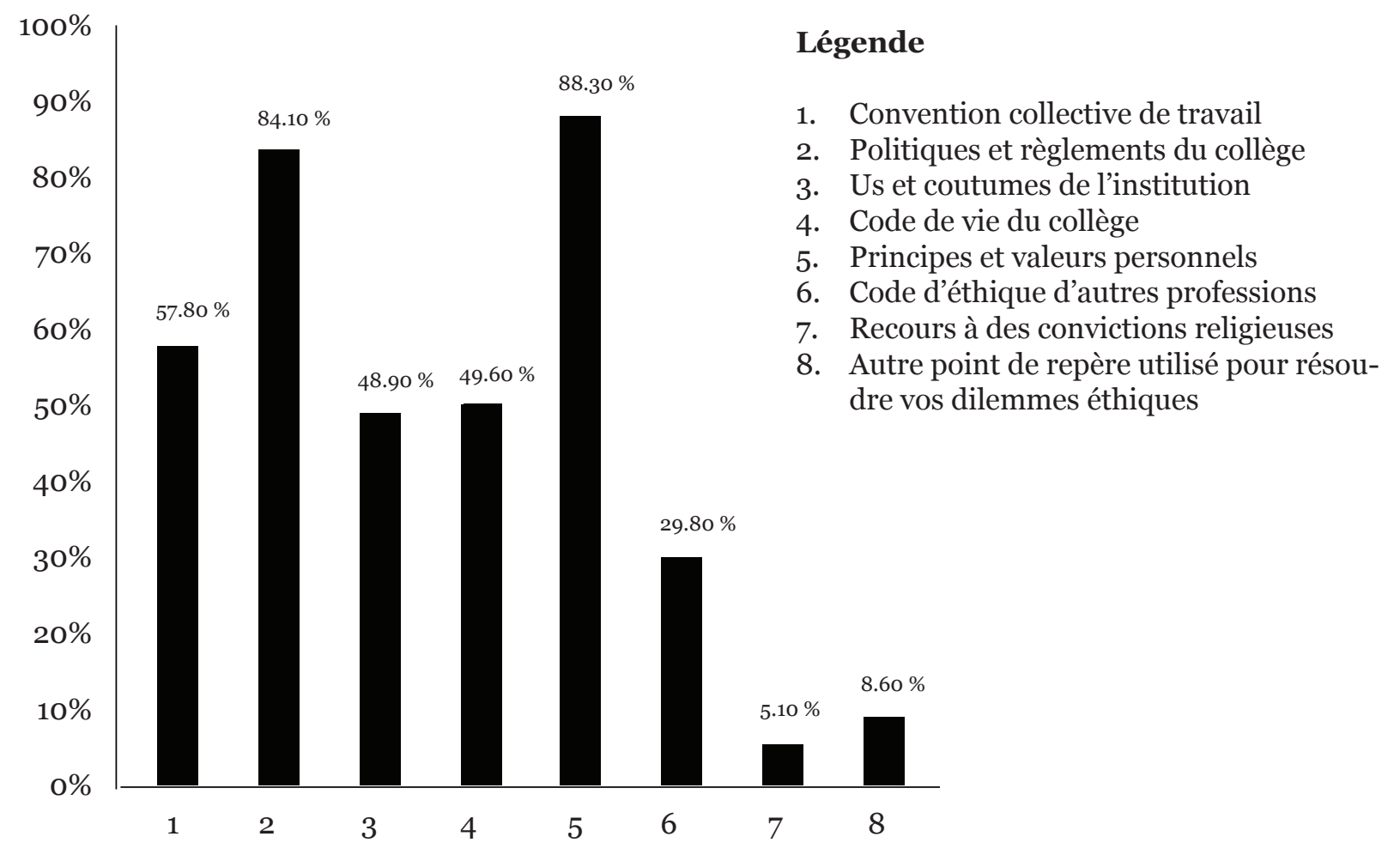

membres de l'administration (51,9\%) et les amis (47,6 \%) jouent aussi un rôle selon de nombreux répondants. De plus, la mise en œuvre d'une procédure de résolution de conflit est vue comme un moyen de négociation ou de discussion avec les personnes concernées par 47,3\% des répondants. L'aspect discussion, peu importe les interlocuteurs, a nettement le dessus sur l'aspect recherche documentaire puisque les lectures (36,3 \%) et la recherche de situations semblables dans les écrits (30\%) sont évoquées par plus ou moins le tiers des répondants. Parmi les autres suggestions rapportées par une minorité $(7,9 \%)$ de répondants, les plus originales ont surtout trait à la pertinence de " lâcher prise » devant le problème éthique ou de le soumettre à un comité d'éthique local.

\section{LA DISCUSSION DES RÉSULTATS}

La comparaison des présents résultats avec les éléments retenus dans le cadre théorique montre une grande convergence entre les deux. Au chapitre des raisons et des indices qui permettent de reconnaître le caractère éthique d'une situation liée à l'enseignement, cas de conscience exigeant la réflexion (Nash, 1996; Young, 1995; Boss, 1998), insatisfaction et malaise devant les dilemmes rencontrés dans l'exercice de sa profession (Campbell, 2008), présence de conflits de valeurs (Mirk, 2005; Norberg et Johansson, 2007) et souci des conséquences sur autrui, sont bien attestés (Brunh et coll., 2002). Toutefois, la première place occupée dans les réponses au questionnaire par les répercussions sur l'intégrité professionnelle de l'enseignant constitue une particularité de cette enquête, 
Figure 3

Les stratégies utilisées pour résoudre des préoccupations éthiqueséthiques

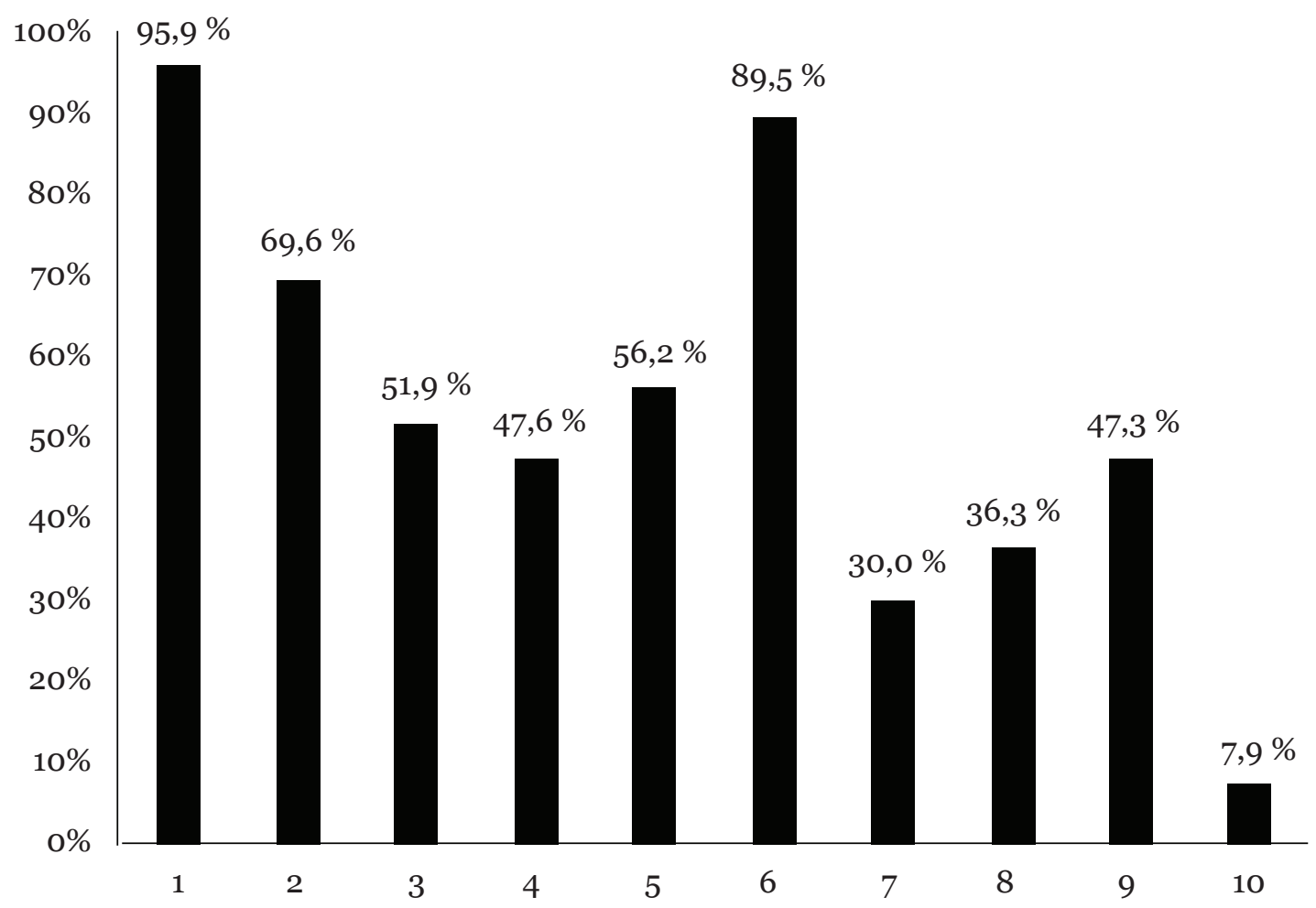

Légende

1. Discussion avec des collègues

2. Discussion avec des étudiants

3. Discussion avec des membres de l'administration

4. Discussion avec des amis

5. Discussion avec des proches autres que des collègues

6. Réflexion personnelle sur le cas

7. Recherche de situations semblables dans les écrits sur le sujet

8. Lectures

9. Utilisation d'une procédure de résolution de conflit

10. Autre stratégie utilisée pour résoudre vos préoccupations d’ordre éthique

car aucun autre auteur n'y fait allusion. L'expression " intégrité professionnelle », choisie par les chercheurs de notre équipe pour rendre compte de certains propos des participants aux groupes de discussion, renvoie au sentiment de bien accomplir leur devoir d'enseignant, d'agir avec probité, en conformité avec leur rôle professionnel et d'éprouver de la satisfaction au regard de la décision prise. Des travaux ultérieurs pourraient permettre de mieux tracer les contours de ce concept et d'en montrer les liens avec celui de professionnalisme.

En ce qui touche les points de repère utilisés pour résoudre des dilemmes éthiques ou pour nourrir la réflexion sur les préoccupations éthiques liées à l'enseignement, la même grande convergence peut être observée entre les constats qui émergent des écrits scientifiques, les résultats de nos groupes de discussion et les résultats de l'enquête. 
Que ces points de repère soient externes ou internes à la personne, à l'instar de ce qu'on peut lire sur la question, les répondants rendent compte d'une tendance à se tourner principalement vers les textes normatifs adoptés par les organisations et les établissements (Campbell, 2000, 2008) ainsi qu'à se fier à leurs propres valeurs (Smith, 1996; Longhi, 1998; Gohier, 2005; Campbell, 2000). Dans les trois ensembles, la référence religieuse est peu présente. Quelques distinctions peuvent toutefois être établies.

Ainsi, si elle est présente dans les écrits scientifiques (Campbell, 2000; Legault, 1997) et mentionnée par les participants aux groupes de discussion (focus groups), la référence aux codes d'éthique ou de déontologie occupe très peu de place dans les réponses à l'enquête. Cela est probablement dû à l'absence de tels codes pour l'enseignement au Québec. En effet, d'une part, l'enseignement n'est pas reconnu comme une profession au sens de la loi et ne relève pas de l'Office des professions du Québec, en plus de n'être pas soumis à l'exigence de formuler un tel code. D'autre part, de fortes réticences par rapport à des codes d'éthique ou de déontologie sont manifestes dans les milieux de l'enseignement. Il convient cependant de noter que la différence entre les résultats de l'enquête et les constats qui émergent de la recension d'écrits peut être atténuée par le fait que chaque collège élabore ses propres politiques et règlements, voire son propre code de vie. La forte proportion de répondants qui disent s'y référer montre en effet que de tels points de repère institutionnels sont importants et utiles pour traiter de cas qui interpellent l'éthique professionnelle.

Une autre différence entre les constats relatifs à la recension des écrits et les résultats de l'enquête tient à la quasi-égalité entre la proportion des répondants qui consultent ces documents institutionnels et ceux qui se fient à leurs valeurs et principes personnels. Cet équilibre entre les repères externes et internes n'est pas souligné dans le corpus des travaux et n'apparaissait pas aussi clairement dans les propos des participants aux groupes de discussion. Quant à l'éclairage éthique que peut fournir la convention collective de travail, aux dires de la moitié des répondants, les écrits consultés n’y font aucune allusion et les participants aux groupes de discussion le mentionnent peu; cet élément constitue certainement une originalité des résultats de cette enquête.

En ce qui concerne les stratégies de résolution des dilemmes éthiques, tout comme dans la revue des écrits (Lafortune et coll., 2008; Strike, 1990; Smith, 1996; Fisch, 1996; Desaulniers, Jutras et Legault, 2005) et dans les propos des participants aux groupes de discussion, ce sont la discussion, surtout celle entre pairs, et la réflexion sur le cas qui dominent. Aucune différence notable entre ces résultats et ce qu'en disent d'autres auteurs n'a été relevée, sauf peut-être en ce qui concerne le silence des répondants à l'enquête vis-à-vis de la stratégie qui consiste à intervenir auprès de collègues fautifs. Toutefois, il est probable que cela soit imputable à la manière dont est construit le questionnaire. En effet, quelques répondants suggèrent la stratégie du lâcher-prise ou du retrait, laquelle a aussi été évoquée par bon nombre de participants des groupes de discussion comme étant particulièrement appropriée lorsque des collègues sont en cause dans une situation de dilemme éthique. Si cette option de réponse avait été offerte explicitement dans le questionnaire, on peut penser qu'elle aurait été retenue par plusieurs répondants. 


\section{CONCLUSION}

Le présent article se proposait de comparer certains résultats de l'enquête menée dans la seconde phase d'un projet de recherche portant sur l'éthique professionnelle de l'enseignement collégial québécois avec ce qu'on trouve dans les écrits scientifiques qui en traitent, ainsi que les résultats de groupes de discussion préalablement dirigés. Il appert que, tant au sujet des raisons et indices évoqués par les enseignants pour reconnaître le caractère éthique de certaines préoccupations professionnelles qu'au sujet des points de repère et des stratégies qu'ils utilisent pour résoudre des préoccupations éthiques, une grande convergence peut être observée. Les raisons qu'ils évoquent pour justifier le caractère éthique d'une situation tiennent surtout à la présence de cas de conscience et de conflits de valeurs ainsi qu'aux conséquences des décisions sur autrui, particulièrement sur les étudiants. Au regard des constats qui émergent de la recension d'écrits et des entrevues de groupe, seule la raison « répercussions sur l'intégrité professionnelle » se démarque et demanderait une exploration plus poussée. Les points de repère qui guident la réflexion des enseignants ayant participé à l'enquête sont semblables à ceux que l'on retrouve dans les écrits scientifiques et à ceux évoqués par les participants aux groupes de discussion. Il s'agit principalement de textes normatifs adoptés par les institutions (repères externes) et de valeurs jugées particulièrement pertinentes par les enseignants (repères internes). Bien qu'à cet égard, les résultats de l'enquête aient notamment permis de mettre en lumière l'importance que revêt la convention collective de travail pour plusieurs répondants, fait qui n'a été relevé par aucun autre auteur jusqu'ici, l'originalité des résultats relève surtout du constat d'un équilibre entre l'utilisation de repères, internes et externes, chez les répondants. Enfin, les stratégies de résolution des préoccupations éthiques les mieux attestées sont identiques, à savoir la discussion entre pairs et la réflexion personnelle. La prochaine étape de nos travaux portera justement sur la manière dont s'effectue la conjonction de ces deux ingrédients, sur le terrain, grâce à une recherche collaborative menée avec des enseignants du collégial. Nous visons à documenter le processus de la délibération éthique.

Enfin, on ne peut conclure sans se questionner sur le faible taux de réponse à cette enquête. Est-ce attribuable aux limites des sondages en ligne et simplement conforme à la décroissance constante des taux de réponse qu'on y observe? Est-ce que la thématique suscite insuffisamment d'intérêt? Ou encore, n'est-ce pas plutôt la preuve qu'Huebner (1996) a raison quand il note la tendance qu'ont les enseignants à n'aborder que les problèmes qu'ils peuvent résoudre techniquement et la difficulté qu'ils éprouvent à parler de leur rôle d'agent moral?*

\section{RÉFÉRENCES}

Academic senate for California community colleges (2002). Faculty as professionals: responsibilities, standards and ethics. [Document téléaccessible à l'adresse http://www. asccc.org/Publications/Papers/Downloads/PDFs/FacultyEthics.pdf] (Consulté le 19 août 2010.)

Baudouin, J. M. (1994). " La réflexion éthique contemporaine », Éducation permanente, vol. 121, pp. 13-52. 
Blais, A. et Durand, C. (2003). « Le sondage ». In B. Gauthier (dir.). Recherche sociale : de la problématique à la collecte de données, 4 éd. (pp. 387-429). Sainte-Foy : PUQ.

Blunden, R. (1996). " Academic loyalties and professional disobedience ». Higher Education research and Development, vol. 15, $\mathrm{n}^{\circ}$ 1, pp. 13-28.

Boss, J. A. (1998). Ethics for life: an interdisciplinary and multicultural introduction. Mountain View, CA : Mayfield.

Bruhn, J.G., Zajac, G., Al-Kazemi, A. et Prescott, L.D. (2002). « Moral positions and academic conduct. Parameters of tolerance for ethics failure ». The Journal of Higher Education, vol. $73, \mathrm{n}^{\circ}$ 4, pp. 461-473.

Buzzelli, C. A. et Johnston, B. (2002). The moral dimensions of teaching: language, power, and culture in classroom interaction. New York and London : Routledge Falmer.

Campbell, E. (2000). "Professional ethics in teaching: towards the development of a code of practice ». Cambridge Journal of Education, vol. 30, nº 2, pp. 203-221.

Campbell, E. (2003). The ethical teacher. Maidenhead, UK : Open University Press, McGraw-Hill.

Campbell, E. (2008). " The ethics of teaching as a moral profession ». Curriculum Inquiry, vol. $38, \mathrm{n}^{\circ} 4$, pp. 357-385.

Couch, S. et Dodd, S. (2005). " Doing the right thing : ethical issues in higher education ». Journal of Family and Consumer Sciences, vol. 97, $\mathrm{n}^{\circ}$ 3, pp. 20-26.

Creswell, J.W. et Plano Clark, V.L. (2007). Designing and conducting mixed methods research. Thousand Oaks, CA : Sage.

Desaulniers, M.-P. (2007). "Enseigner au collégial, une profession à partager ». Pédagogie collégiale, vol. 20, $\mathrm{n}^{\circ}$ 3, pp. 5-11.

Desaulniers, M.-P. et Jutras, F. (2006). L'éthique professionnelle en enseignement. Fondements et pratiques. Québec : PUQ.

Desaulniers, M.P., Jutras, F. et Legault, G.A. (2005). « Les enjeux de la compétence éthique dans la formation des enseignants ». In Gohier, C. et Jeffrey, D. (dir.), Enseigner et former à l'éthique (pp.131-147). Saint-Nicolas : PUL.

Desautels, L. (2005). Une approche d'éthique appliquée dans les cours de philosophie éthique au collégial : présence et incidence sur l'intérêt et la réussite des élèves. Thèse présentée à l'Université Laval dans le cadre d'un programme offert à l'Université de Sherbrooke en vertu d'un protocole d'entente avec l'Université Laval.

Desautels, L., Gohier, C., Jutras, F. (2009). Les préoccupations éthiques d'enseignants du réseau collégial francophone au Québec. Revue canadienne de l'éducation, vol. 32, $\mathrm{n}^{\mathrm{o}} 3,395-419$.

Fisch, L. (1996). «Toward more ethical teaching ». In Fisch, L. (Ed.) Ethical dimensions of college and university teaching: understanding and honoring relationship between teachers and students (pp. 89-99). San Francisco : Jossey Bass Publishers.

Fortin, P. (1995). La morale L'éthique L'éthicologie. Sainte-Foy: PUQ. 
Gohier, C. (2005) « La formation des maîtres et l'orientation de la conduite humaine, un art entre éthique et déontologie », In C. Gohier et D. Jeffrey (dir.), Enseigner et former à l'éthique (pp. 41-60). Sainte-Foy : Presses de l'Université Laval.

Gohier, C., Jutras, F. et Desautels, L. (2007a). « Mise au jour d'enjeux éthiques de la profession enseignante au collégial ». Pédagogie collégiale, vol. 20, $\mathrm{n}^{\circ}$ 2, pp. 30-35.

Gohier, C., Jutras, F. et Desautels, L. (2007b). La mise au jour des enjeux éthiques de la professionenseignante: lefocus group, une bonne méthode? In Actes du congrès international Actualité de la Recherche en Éducation et en Formation (AREF), [Document téléaccessible à l'adresse [http: www.congresintaref.org $\backslash$ actes_site.php], 10 pages et CD-ROM.

Gohier, C., Desautels, L., Jutras, F. (2010a). Les préoccupations éthiques chez les enseignants de l'ordre collégial : caractéristiques, points de repère et stratégies de résolution. Revue des sciences de l'éducation, vol. 36, $\mathrm{n}^{\circ}$ 1, 213-231.

Gohier, C., Desautels, L., Joly, J., Jutras, F., Ntebutse, J.G. (2010b). Les préoccupations éthiques des enseignants de l'ordre collégial: une enquête en ligne. McGill Journal of Education, vol. 45, $\mathrm{n}^{\circ}$ 3, 1-22.

Gouvernement du Québec (2001). La formation à l'enseignement. Les orientations. Les compétences professionnelles. Québec : Ministère de l'Éducation.

Habermas, J. (1991). De l'éthique de la discussion. Paris : Flammarion.

Hamilton, N. (2006). « Faculty professionalism: Failures of socialization and the road to loss of professional autonomy ». Liberal Education, vol. 92, ${ }^{\circ}$ 4, pp. 14-21.

Hansen, D. T. (2001). " Teaching as a moral activity ». In V. Richardson (Ed.), Handbook of research on teaching, $4^{\mathrm{e}}$ éd. (pp. 826-857). Washington, DC : American Educational Research Association.

Hanson, K. (1996). « Between apathy and advocacy: teaching and modeling ethical reflexion ». New Directions for Teaching, vol. 66, pp. 33-36.

Hardy, D.E. (2002). «Ethical considerations affecting teaching in community colleges: an abundance of feelings and limited facts ». Community College Journal of Research and Practice, vol. 26, $\mathrm{n}^{\circ}$ 5, pp. 383-399.

Huebner, D. (1996). «Teaching as a moral activity ». Journal of Curriculum and Supervision, vol. 11, $\mathrm{n}^{\circ}$ 3, pp. 267-275.

Jackson, P. W., Boostrom, R. E. et Hansen, D. T. (1993). The moral life of schools. San Francisco : Jossey-Bass.

Jutras, F., Joly, J. et Legault, G.A. (2005). « L’intervention professionnelle en enseignement : les conceptions de la profession chez le personnel enseignant du primaire et du secondaire ». Revue des sciences de l'éducation, vol. 31, ${ }^{\circ}$ 3, pp. 563-583.

Keith-Spiegel, P., Wittig, A.F., Perkins, D.V., Ware Balogh, D. et Whitley, B.E. (1996). «Intervening with colleagues ». In L. Fisch (Ed.). Ethical dimensions of college and university teaching: understanding and honoring the special relationship between teachers and students (pp. 75-78). San Francisco : Jossey-Bass Publishers. 
Knight, J. et Auster, C.J. (1999). « Faculty conduct : an empirical study of ethical activism ». The Journal of Higher Education, vol. 70, n² 2, pp. 188-210.

Lafortune, L., Thibodeau, S., Ticon, W. et Walther, É. (2008). « Développer et évaluer la compétence éthique en formation à l'enseignement ». In Réfléchir pour évaluer des compétences professionnelles à l'enseignement. Deux regards, l'un québécois, l'autre suisse (pp. 240-256). Québec : PUQ.

Legault, G.A. (dir.) (1997). Enjeux de l'éthique professionnelle Tome II. L'expérience québécoise. Sainte-Foy : Presses de l’Université du Québec.

Legault, G.A. (1999). Professionnalisme et délibération éthique. Québec : Presses de l’Université du Québec.

Longhi, G. (1998). Pour une déontologie de l'enseignement. Paris : ESF éditeur.

Lozar Manfreda, K., Bosnjak, M., Berzelak J., Haas, I. et Vehovar V. (2008) «Web surveys versus other survey modes: a meta-analysis comparing response rates ", International Journal of Market Research, vol. 50, n ${ }^{\circ}$, pp. 79-104.

MacFarlane, B. (2002). " Dealing with Dave's dilemmas: exploring the ethics of pedagogic practice ». Teaching in Higher Education, vol. 7, $\mathrm{n}^{\circ}$ 2, pp. 167-178.

MacFarlane, B. (2004). Teaching with integrity: the ethics of higher education practice. Abington, Oxfordshire : RouteledgeFarmer.

Massé, R. et Saint-Arnaud, J. (2003). Éthique et santé publique. Québec : PUL.

Mirk, P. (2005). « Ethical literacy for today's schools ». Education Canada, vol. 45, $\mathrm{n}^{\mathrm{o}} 3$, pp. 17-19.

Moreau, D. (2003). La construction de l'éthique professionnelle des enseignants au cours de leur formation et leur entrée dans le métier : la genèse d'une éthique appliquée de l'éducation. Lille : Atelier national de reproduction des thèses.

Moreau, D. (2007). "L'éthique professionnelle des enseignants : déontologie ou éthique appliquée de l'éducation? » Les sciences de l'éducation pour l'ère nouvelle, vol. $40, \mathrm{n}^{\circ} 2$, pp. 53-75.

Munoz-Leiva F., Sánchez-Fernández, J., Montoro-Rios, F. et Ibáñez-Zapata, J. (2009). "Improving the response rate and quality in web-based surveys through the personalization and frequency of reminder mailings ». Quality and Quantity, pp. 10371052.

Murray, H., Gillese, E., Lennon, M., Mercer, P. et Robins, M. (1996). In L. Fisch (Ed.). Ethical dimensions of college and university teaching : understanding and honoring the special relationship between teachers and students (pp. 57-63). San Francisco : JosseyBass Publishers.

Nash, R. J. (1996). "Real world" ethics: frameworks for educators and human service professionals. New York : Teachers College Press.

Norberg, K. et Johansson, O. (2007). « Ethical dilemmas of swedish school leaders. » Educational Management Administration \& Leadership, vol. 35, n 2, pp. 277-294. 
Ordre des enseignantes et des enseignants de l'Ontario (2006). Normes de déontologie de la profession enseignante. [Document téléaccessible à l'adresse <http://www.oct.ca/ standards/ethical_standards.aspx?lang=fr-CA>.] (Consulté le 19 août 2010.)

Parizeau, M.-H. (1994). «Éthique et éthiques appliquées : l'émergence des théories composites », Philosopher, vol. 16, pp. 133-143.

Prairat, E. (2005). De la déontologie enseignante. Paris : PUF.

Richardson, V. et Fenstermacher, G. D. (2001). " Manner in teaching: the study in four parts ». Journal of Curriculum Studies, vol. 33, n 6, pp. 631-637.

Ricœur, P. (1991). Lectures 1. Autour du politique. Paris : Seuil.

Sheehan, K. (2001). « E-mail survey responses rates : a review ». Journal of computerMediated Communication, vol. $6, \mathrm{n}^{\circ}$ 2. [Document téléaccessible à l'adresse < http:// jcmc.indiana.edu/vol6/issue2/sheehan.html >.] (Consulté le 18 avril 2001.)

Shih, T-H. et Fan, X. (2008). « Comparing response rates from web and mail surveys: a meta-analysis ». Field Methods, vol. 20, n 3 , pp. 249-271.

Smith, D.C. (1996). « The ethics of teaching ». In Fisch, L. (Ed.). Ethical dimensions of college and university teaching : understanding and honoring relationship between teachers and students. (pp. 5-14) San Francisco : Jossey Bass Publishers.

Strike, K.A. (1990). " The legal and moral responsibility of teachers ». In Goodlad, J., Soder, R. et Sirotnik, K. (dir.) The moral dimensions of teaching (pp. 188-223). San Francisco et Oxford : Jossey-Bass Publishers.

Watson, D. (2007). " Does higher education need a hippocratic oath? " Higher Education Quarterly, vol. 61, $\mathrm{n}^{\circ}$ 3, pp. 362-374.

Young, D. (1995). "Understanding ethical dilemmas in education ». Educational Horizons, vol. $74, \mathrm{n}^{\circ}$ 1, pp. 37-42.

\section{REMERCIEMENTS}

Les auteurs remercient le Conseil de recherche en sciences humaines du Canada pour le financement de la recherche (410-2006-2376) ainsi que Marianne St-Onge, Vincent Beaucher, Simon Lavoie, Mamadou Bhoye Bah et Mélanie Grenier pour leur collaboration à titre d'assistants de recherche.

\section{CONTACT INFORMATION}

Luc Desautels

L'Assomption (Québec)

luc.desautels@collanaud.qc.ca

Luc Desautels est enseignant de philosophie au collégial à L'Assomption (Québec) et coordonnateur régional de la recherche au Cégep régional de Lanaudière. Détenteur d'une maîtrise ès arts en théologie (Collège universitaire dominicain, Ottawa), d'une maîtrise en éducation (Université de Montréal) ainsi que d'un doctorat en philosophie (Université de Sherbrooke), il s'intéresse particulièrement aux applications pédagogiques 
des technologies de l'information, aux questions d'éthique appliquée et à l'amélioration continue de la qualité en éducation.

Christiane Gohier est professeure titulaire au Département d'éducation et pédagogie à l'Université du Québec à Montréal et chercheure au Centre interdisciplinaire sur la formation et la profession enseignante (CRIFPE). Ses recherches et publications portent sur l'éthique en éducation, la construction d l'identité professionnelle des enseignants et des futurs enseignants, les fondements de l'éducation, particulièrement l'épistémologie et les finalités de l'éducation. On trouve une liste de ses travaux en accédant au http:// ww.er.uqam.ca/nobel/r17101.

Détenteur d'un doctorat en psychologie (Ph.D.) depuis 1981, Jacques Joly est professeur au Département de psychoéducation de l'Université de Sherbrooke depuis 2000. Il y a enseigné les méthodes statistiques, la méthodologie scientifique et l'évaluation de programme. Il est présentement vice-doyen aux études supérieures en recherche et secrétaire de faculté de la Faculté d'éducation, où il assume toujours des tâches d'enseignement dans le programme de doctorat en éducation. Ses recherches portent principalement sur l'implantation de programmes de prévention ou d'intervention en milieu scolaire ou dans les services sociaux.

Docteure en philosophie de l'éducation et professeure titulaire en Fondements de l'éducation au Département de pédagogie de l'Université de Sherbrooke, France Jutras s'intéresse au rôle des valeurs personnelles, professionnelles et sociales dans le développement professionnel des enseignants. Son cadre de référence se situe dans une perspective d'éthique appliquée et d'éducation à la citoyenneté. Elle est membre de l'IRPÉ et de l'ERGÉA de l'Univertsité de Sherbrooke.

Jean Gabin Ntebutse détient un doctorat en pédagogie universitaire de l'Université de Sherbrooke. Après un stage postdoctoral à l'Université du Québec à Montréal, il est devenu professeur à l'Université de Sherbrooke. Ses intérêts de recherche ont trait notamment au changement de paradigme dans la pédagogie universitaire, à l'éthique en enseignement supérieur, ainsi qu'à l'apprentissage et au développement. 